\title{
Autotoma para detección de VPH comparada con muestra convencional en el tamizaje de cáncer cervicouterino
}

\author{
DOI 10.5377/alerta.v5i1.12806
}

Silvana Nazaré Cabrera Saca ${ }^{1 *}$, Astrid Leonor Linares García ${ }^{2}$, Javier Ernesto Martínez Meléndez ${ }^{3}$, Emilio Jacobo Abullarade Navarrete ${ }^{4}$

1-4. Universidad Dr. José Matías Delgado, Antiguo Cuscatlán, El Salvador

${ }^{*}$ Correspondencia

$\checkmark$ silna-95@hotmail.com

1. (1) 0000-0002-5835-6128

2. (1) 0000-0003-0713-0134

3. (1) 0000-0003-2520-9313

4. (1) 0000-0003-4898-032X

\begin{abstract}
Resumen
El cáncer de cuello uterino es la cuarta causa de mortalidad por cáncer en mujeres; $88 \%$ de los casos provienen de países en vías de desarrollo debido al escaso acceso al tamizaje y tratamiento. Debido a esto, en los países de bajo desarrollo humano estrategias como la autotoma para la detección del virus del papiloma humano han adquirido gran importancia en la actualidad, aumentando hasta en un $45 \%$ el tamizaje en estos países. La presente revisión bibliográfica tiene como objetivo determinar si existen diferencias entre técnica convencional para detección del virus del papiloma humano y la autotoma en países de mediano y bajo desarrollo humano, así como las ventajas y limitantes que esta presenta. Se realizó la búsqueda de la literatura mediante las bases de datos PubMed, Google Scholar y SciELO, empleando artículos originales, ensayos clínicos, de revisión, entre otros. Se demostró que la autotoma para la detección del virus del papiloma humano tiene una especificidad similar a la toma convencional por parte de personal médico y que incrementa el tamizaje, ya que es más aceptada que la muestra tomada por el clínico debido a que facilita el acceso y aborda las limitantes socioculturales percibidas por las usuarias.

\section{Palabras clave}

Infección por VPH, cáncer de cuello uterino, papiloma.
\end{abstract}

\begin{abstract}
Cervical cancer is the fourth cause of mortality by cancer in women. $88 \%$ of all cases come from developing countries, in which access to screening and treatment is scarce. For this reason in low-developed countries approaches like the self-sampling human papillomavirus detection tests have acquired high relevance at the moment, increasing screening by up to $45 \%$ in these countries. The present review aims to determine if there are differences between self-sampling and traditional sampling taken by health care providers while screening for human papillomavirus in the middle to low development countries as well as its advantages and limitations. The literature search was carried out through databases, PubMed, Google Scholar, SciELO, using original articles, clinical trials, review articles, among others. it is shown that human papillomavirus self-sampling has a specificity similar to traditional sampling taken by health care providers, it increases the uptake of screening because is more accepted due to its easier access and that it addresses the sociocultural factors perceived by the patients.
\end{abstract}

Keywords

Human Papillomavirus Infection, uterine cervical neoplasms, Papilloma.

\section{Introducción}

La infección por genotipos de alto riesgo (16 y 18) del virus del papiloma humano (VPH) se asocian a $80 \%$ de los casos de cáncer cervicouterino invasivo!
En países en desarrollo, la sobrevida se ve influenciada por factores socioeconómicos, culturales, el difícil acceso a programas de tamizaje y tratamiento. Para el año 2018 se reportaron 570000 casos y más de 311000 muertes por cáncer cervicouterino a nivel mundial, siendo este el cuarto cáncer más
ACCESO ABIERTO

Self-sampling HPV detection test against conventional sampling in cervical cancer screening

Citación recomendada:

Cabrera Saca SN, Linares

García AL, Martínez Meléndez

JE, Abullarade Navarrete EJ.

Autotoma para detección de

VPH comparada con muestra convencional en el tamizaje de cáncer cervicouterino. Alerta. 2022;5(1):81-87. DOI: 10.5377/ alerta.v5i1.12806

Recibido:

26 de octubre de 2021

Aceptado:

23 de diciembre de 2021.

Publicado:

27 de enero de 2022

Contribución de autoría: SNCS' ${ }^{1}$, ALLG $^{2}$, JEMM ${ }^{3}$, EJAN 4 : contribución sustancial a la concepción, diseño del trabajo, redacción y revisión crítica del artículo.

\section{Conflicto de intereses:} Los autores declaran no tener conflictos de interés en el estudio. 
diagnosticado y la cuarta causa de mortalidad por cáncer en la población femenina'. El $84 \%$ de los casos y el $88 \%$ de las muertes provienen de países con mediano y bajo Índice de Desarrollo Humano². En particular, para el año 2020 en El Salvador se reportaron 530 nuevos casos y 317 muertes ocasionadas por cáncer cervicouterino, siendo el segundo cáncer más diagnosticado en mujeres ${ }^{3}$.

La Organización Mundial de la Salud (OMS) en su «Estrategia global para eliminar el cáncer cervicouterino como problema de salud pública» propone vacunar contra VPH al $90 \%$ de las niñas a los 15 años, tamizar el $70 \%$ de las mujeres a los 35 y 45 años y tratar el $90 \%$ de los casos identificados 4 .

Para el año 2030, en los países de mediano y bajo desarrollo humano se estima que la vacunación tendrá mínimo efecto en la mortalidad por cáncer cervicouterino, con una reducción estimada de 0,1 \% de la mortalidad, mientras que el tamizaje con prueba de VPH y tratamiento reducirá la mortalidad en un 34,2\%, previniendo hasta aproximadamente unas 400000 muertes $^{5}$. La cobertura actual del tamizaje de cáncer cervicouterino en los países en desarrollo alcanza solamente un promedio del 19,0 \% ${ }^{6}$. Sin embargo, Arrossi et al. encontraron un aumento de la cobertura de tamizaje del $45 \%$ ( $p<0,001)$ al implementar la autotoma de la prueba de VPH como alternativa de tamizaje a la prueba recolectada por personal médico?.

Por lo que el objetivo de este estudio es determinar si existen diferencias en términos de aceptabilidad, sensibilidad, especificidad y concordancia de resultados en la técnica por autotoma de muestra vaginal para la detección del virus del papiloma humano en comparación con la toma por personal de salud como estrategia de tamizaje para el cáncer cervicouterino en países de mediano y bajo desarrollo humano.

\section{Desarrollo}

La autotoma del VPH es un método de tamizaje realizado por la misma usuaria desde su lugar de residencia al recibir un kit especializado donde se obtiene una muestra cervical o vaginal para determinar la presencia del virus ${ }^{8}$. Por lo general, los materiales del kit para la autotoma incluyen el cepillo, tubo colector, una guía instructiva, información sobre los posibles resultados que se obtienen, fichas de solicitud de tamizaje al laboratorio, marcadores de identificación para el tubo colector, carnet que brinda un registro de la prueba realizada y un documento sobre la prevención del cáncer cervicouterino9. La principal diferencia que presenta respecto a la técnica convencional es que la autotoma no requiere colocación de espéculo para obtener la muestra.

Para su realización, la usuaria adquiere una posición cómoda, ya sea en cuclillas o donde las rodillas se flexionen y los glúteos desciendan a nivel de los talones. Ella introduce el cepillo de 7 a 10 centímetros a través de la vagina hasta el orificio cervical para realizar tres giros de $360^{\circ}$ en $10-30$ segundos; posteriormente, se coloca la muestra dentro del tubo colector y se sella para ser entregado al establecimiento de salud. Sin embargo, se han descrito otras técnicas para recolectar la muestra que varían según el kit que se ha utilizado ${ }^{10}$ (Figura 1).

Una de las ventajas que presenta la autotoma es una mayor aceptabilidad y participación en el tamizaje de las usuarias en comparación con programas que no incluyen la autotoma. Específicamente, Peeters et al. describieron en su estudio que un 78\% de las participantes entre 25-64años que no se habían realizado su citología en los últimos tres años, accedieron a participar en el tamizaje de cáncer cervicouterino cuando se les ofreció el kit de autotoma, en comparación a un $51 \%$ que prefirieron la técnica realizada por el personal médico ${ }^{11}$. Asimismo, Buist et al. evidenciaron un incremento de $80 \%$ en el tamizaje con el envío de kits para autotoma a los domicilios en mujeres que no se tamizaban con el método convencional, logrando disminuir el número de consultas a los establecimientos de salud, así como las barreras financieras, geográficas y personales para el tamizaje ${ }^{10}$.

Otra ventaja de la autotoma con respecto a la técnica convencional es que las usuarias refieren menos dolor, vergüenza y más comodidad. Por ejemplo, Kamath et al. en su metanálisis establecieron que 75-97 \% de las participantes consideraron que la autotoma fue fácil de realizar y a un 60-90\% le pareció indoloro y menos vergonzoso; además, se demostró que este método de tamizaje fue preferido sobre el recolectado por el personal médico en 57-100\% de los casos $^{12}$.

Por otro lado, se ha demostrado que la precisión de la autotoma en detectar lesiones precancerosas de alto grado es similar a la de muestras recolectadas por el clínico cuando se utiliza reacción en cadena de la polimerasa (PCR) como método para procesar las muestras ${ }^{13}$. Sin embargo, tiene la desventaja que se necesita una organización compleja para suministrar los kits, requiere un método de transporte adecuado de las muestras, educar a la 

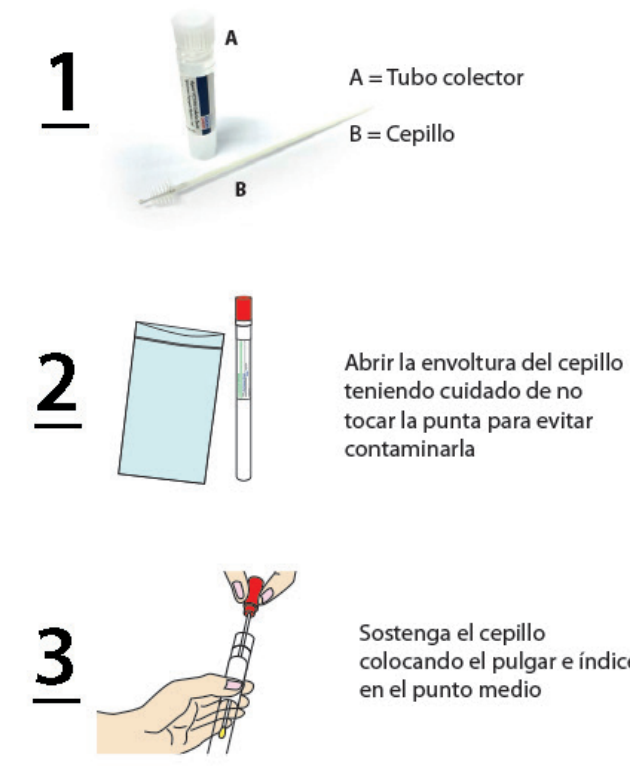

Sostenga el cepillo colocando el pulgar e índice en el punto medio

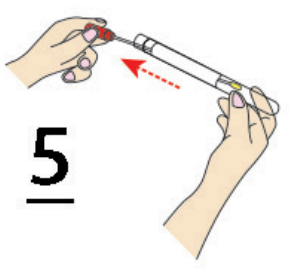

Desenrosque la tapa del tubo colector e inmediatamente coloque la muestra obtenida con el cepillo hasta el medio de cultivo

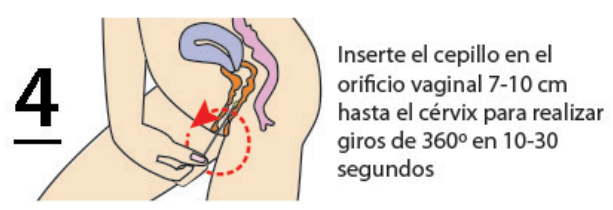

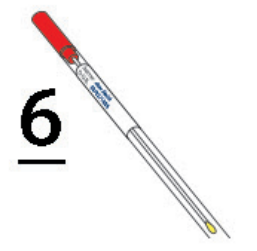

Encuentre la línea de corte de cepillo y rompa en este punto contra el lado opuesto del tubo. Luego deseche la parte superior restante

Figura 1. Pasos para la obtención de muestra por autotoma para prueba VPH. Elaboración propia.

población sobre la técnica y resultados y un sistema de seguimiento adecuado para dar tratamiento a las pacientes que tienen resultados positivos ${ }^{14}$.

\section{Concordancia, sensibilidad y especificidad de ambas técnicas de obtención de muestra en pruebas de detección}

Con respecto a la prueba de $\mathrm{VPH}$ basada en amplificación de señal (AS), se encontró una concordancia moderada de resultados entre las muestras obtenidas por autotoma comparada con la técnica convencional. En particular, De Melo et al. en su estudio aleatorizado de 204 mujeres brasileñas, compararon los resultados de la prueba de VPH utilizando autotoma y la técnica convencional de obtención de muestra, encontrando una concordancia moderada de $\mathrm{k}$ (índice Kappa) de 0,43 (95 \% IC 0,27$0,59)^{15}$.

De igual forma, el ensayo clínico realizado por Ajenifuja et al. en 194 mujeres nigerianas mostró una concordancia moderada con un índice $k$ de 0,47 ( $p<0,05)$ entre los resultados de ambas técnicas ${ }^{16}$. Una concordancia de resultados similar ( $\mathrm{K} 0,55,95 \%$ IC 0,45$0,65)$ fue obtenida por Senkomago et al. en su estudio de cohortes en 350 mujeres trabajadoras del sexo en Kenia ${ }^{17}$.

En contraste, al utilizar pruebas para VPH basadas en PCR, la concordancia entre resultados de muestra obtenida por el clínico y autotoma fue alta, específicamente en los estudios de cohortes realizados por Esber et al. ${ }^{18}$, Obiri-Yeboah et al. ${ }^{19}$ y Kuriakose et al. ${ }^{20}$. En estos encontraron índices $\mathrm{k}$ de concordancia altos entre los resultados de ambos tipos de muestras, de 0,83 (95\% IC 0,71-0,96), 0,88 ( $\leq \leq 0,001)$ y 0,82 ( $p<0,001)$, respectivamente. Similarmente, Wong et al. ${ }^{21}$, Cremer et al..22, y Toliman et al. ${ }^{23}$ reportaron concordancias altas de resultados para prueba de VPH: k 0,74 (95 \% IC 0,41-1,00), k 0,70 (95 \% IC 0,68-0,73) y k 0,74-0,77 (95 \% IC, 0,70-0,83).

En cuanto a la sensibilidad de la prueba de VPH utilizando autotoma fue evaluada por Kelly et al. en su metanálisis que incluyó 11 estudios para detectar neoplasia intraepitelial cervical grado 2 o mayor (NIC2+) utilizando PCR; encontrándose una sensibilidad inferior para muestra recolectada por autotoma (73,6 \%), en contraste con la muestra recolectada por técnica convencional $(88,1 \%)^{24}$.

Por otro lado, en su metanálisis basado en 81 estudios, Arbyn et al. no encontraron mayor diferencia entre la sensibilidad (96\%) y especificidad (79\%) de ambos tipos de muestras en pruebas de VPH al utilizar PCR. Mientras que al utilizar pruebas basadas en AS se evidenció una sensibilidad inferior (77 \%) para detectar neoplasia intraepitelial cervical grado 2 o mayor ( $\mathrm{NIC2}+$ ) en muestras obtenidas por autotoma, comparada con la muestra tomada por el personal médico 
(93\%) y especificidades similares (84 vs. $86 \%)^{25}$.

De Melo et al. también reportaron una menor sensibilidad (50\%) para células atípicas de significancia indeterminada o mayor (ASCUS+) en muestras obtenidas por autotoma de lavado vaginal utilizando AS comparada con la recolectada por el clínico $(81,5 \%)^{21}$. Así mismo, Senkomago et al. encontraron una menor sensibilidad de la autotoma (79\%) para identificar lesión intraepitelial de alto grado o mayor (LIE-AG+) en comparación con la muestra colectada por el personal médico $(86 \%)^{23}$.

No obstante, al utilizar pruebas de VPH basadas en PCR (Xpert y Cobas 4800), Toliman et al. determinaron una sensibilidad para LIE-AG+ de muestras por autotoma (91,7\% y 88,9\%) comparables al estándar clínico $(91,7 \%)^{23}$. Viviano et al. también reportaron una mayor sensibilidad (83,3\%) para LIE-AG+ a los 12 meses en mujeres que recibieron termoablación utilizando PCR en muestras obtenidas por autotoma en comparación con muestra convencional, $71,4 \%$.

\section{Factores socioculturales que influyen en la aceptabilidad de ambas técnicas}

Existen diversas barreras que dificultan e imposibilitan el acceso a servicios de tamizaje de cáncer cervicouterino en las mujeres de los países de mediano y bajo desarrollo humano. Por ejemplo, Buchanan et al., en el año 2017, describieron en una población de 60 mujeres de Kenia que no se tamizaban debido al temor de obtener resultados positivos, ya que consideraban el diagnóstico como una «sentencia de muertes ${ }^{27}$. Similarmente, Ampofo et al. encontraron que la posibilidad de tener cáncer cervicouterino causaba temor al $80 \%$ de las mujeres $(p<0,001)^{28}$.

Gottschlisch et al. reportaron que los factores económicos y culturales también limitan a las mujeres a realizarse el tamizaje cervicouterino, encontrando que en una población de 15317 mujeres en Guatemala, el $60 \%$ no se tamizó debido a presentar problemas económicos que les impedían tener acceso al tamizaje ( $p<0,001) ; 35 \%$ por la larga distancia que debían recorrer para asistir a los establecimientos de salud ( $p<0,001)$ y $20 \%$ por falta de permiso por parte de la pareja para realizarse el tamizaje por un personal médico $(p<0,001)^{29}$.

Esto hallazgos coinciden con lo descrito por Gizaw et al., quienes reportaron que en una población de 324 mujeres etíopes, $20 \%$ de estas refirió que no se realizaba el tamizaje por no contar con el apoyo de su pareja. Además, se describe que las mujeres del área rural tienen el doble de posibilidad de no realizarse el tamizaje en comparación con las que viven en zonas urbanas $(A O R=2$; $95 \% \mathrm{Cl}, 1,1-3,5)^{30}$

Los conocimientos sobre el cáncer cervicouterino inciden sobre la decisión de tamizarse. Específicamente, Rashid et al. encontraron en una población de estudiantes universitarios en India, que solo $64,5 \%(p<0,001)$ respondió conocer la enfermedad y 33,5\% ( $p<0,001)$ conocía su relación con el VPH ${ }^{31}$. Asimismo, Akinlotan et al. reportaron en una población sin acceso a seguro médico en Estados Unidos que 19,8 \% (OR 1,99, 95 \% IC 1,16-3,44) de hispanos y el 27,2\% (OR 2,06, 95 \% IC 1,15-3,68) de afroamericanos identificaban la falta de conocimiento sobre la enfermedad como una barrera para el tamizaje de cáncer cervicouterino ${ }^{32}$.

De igual manera, Mukama et al. encontraron en una población de 900 mujeres de Uganda que solo 45,7 \% tenía conocimientos sobre una o más modalidades de tamizaje para cáncer cervicouterino y solo el 0,01 \% conocía la frecuencia recomendada de tamizaje ${ }^{33}$.

Con respecto a las barreras identificadas para el tamizaje mediante la muestra por personal médico, Laskow et al. encontraron que de las mujeres que prefirieron la autotoma, $44 \%$ respondió sentirse incómoda o avergonzada durante el examen pélvico; $39 \%$ no quería ser evaluada por un hombre y $24 \%$ no quería que se le colocase espéculo debido a incomodidad ${ }^{34}$. Además, Alfaro et al. encontraron que ser atendida por personal masculino $(71,4 \%)$, falta de comodidad o vergüenza $(64,3 \%$ ) y la expectativa de dolor, eran motivos por los cuáles las mujeres no habían acudido al tamizaje ${ }^{35}$.

Yeh et al. en un metanálisis encontraron que las mujeres eran más proclives a tamizarse por medio de autotoma en comparación con la muestra tomada por personal de salud (RR: 2,13, 95 \% IC 1,89-2,40) ${ }^{36}$. En una población de mujeres nicaragüenses, Quincy et al. reportaron un índice de aceptabilidad de la autotoma mayor que la muestra convencional $(18,40 \pm 2,73$ vs. $17,56 \pm 2,92$, $p<0,001$.

La autotoma también incrementa el número de mujeres tamizadas. En particular, Murchland et al. encontraron en 956 mujeres de las localidades de Santiago de Atitlán e Izabal, en Guatemala, que $93,4 \%$ y $62,4 \%$ ( $p<0,001$ ) aceptarían tamizarse mediante autotoma. Además, de las mujeres que se tamizaron en ambas poblaciones, un 98,1 \% y 95,3\% dijeron que lo harían de nuevo ${ }^{37}$. 


\section{Conclusiones}

La autotoma de muestra para la detección del VPH permite una mayor cobertura del tamizaje para cáncer cervicouterino, ya que aumenta la participación de las mujeres, facilita el acceso y disminuye la cantidad de consultas en los servicios de salud y barreras geográficas. Sin embargo, su empleo requiere de una organización compleja para el suministro de kits y un seguimiento adecuado de los casos positivos.

Se encontró que existe una concordancia y especificidad similares entre los resultados por autotoma y los obtenidos mediante la muestra tomada por personal médico para prueba de VPH basada en PCR. La autotoma fue menos sensible que la muestra tomada por el clínico al utilizar AS.

Se identificaron como principales factores socioculturales que afectan el tamizaje cervicouterino la vergüenza durante el examen ginecológico, la falta de aprobación de la pareja, el miedo al cáncer, así como la falta de información sobre la enfermedad y su tamizaje. La autotoma fue más aceptada que la muestra tomada por el clínico y aumentó la cantidad de mujeres que fueron tamizadas.

Se encontraron como principales diferencias entre la autotoma y la muestra tomada por el clínico, el uso de espéculo, quién toma la muestra, la posición que adopta la usuaria, una menor sensibilidad de la autotoma cuando la muestra es procesada por métodos de AS y una mayor aceptabilidad y participación de las usuarias hacia la autotoma. La principal similitud es que las muestras obtenidas por ambas técnicas se procesan de la misma forma; la concordancia, especificidad y sensibilidad de ambas técnicas son semejantes cuando se utiliza PCR.

\section{Financiamiento}

No hubo financiamiento para la elaboración de este manuscrito

\section{Referencias Bibliográficas}

1. Bray F, Ferlay J, Soerjomataram I, Siegel RL, Torre LA, Jemal A. Global cancer statistics 2018: GLOBOCAN estimates of incidence and mortality worldwide for 36 cancers in 185 countries. CA Cancer J Clin. 2018 Nov;68(6):394-424. DOI: 10.3322/caac.21492

2. Arbyn $M$, Weiderpass E, Bruni L, de Sanjosé S, Saraiya M, Ferlay J, et al. Estimates of incidence and mortality of cervical cancer in 2018: a worldwide analysis. The Lancet Global
Health. 2020 8(2):191-203. DOI: $10.1016 /$ S2214-109X(19)30482-6

3. Organización Mundial de la Salud. El Salvador Data Fact Sheets. 2021. Fecha de consulta: 8 de septiembre de 2021. Disponible en: https://gco.iarc.fr/today/data/factsheets/ populations/222-el-salvador-fact-sheets.pdf

4. Organización Mundial de la Salud. Global strategy towards eliminating cervical cancer as a public health problem. Geneva: $\mathrm{WHO}$; 2020. Fecha de consulta: 8 de septiembre de 2021. Disponible en: https://www.who.int/ publications-detail-redirect/9789240014107

5. Canfell K, Kim J, Brisson M. Mortality impact of achieving WHO cervical cancer elimination targets: a comparative modelling analysis in 78 low-income and lower-middle-income countries. Lancet 2020; 395: 591-603. DOI: 10.1016/S0140-6736(20)30157-4

6. Gakidou E, Nordhagen S, Obermeyer Z. Coverage of Cervical Cancer Screening in 57 Countries: Low Average Levels and Large Inequalities. PLOS medicine. Jun 2008. 5(6):863-8. DOI: 10.1371/journal. pmed.0050132

7. Arrossi S, Paolino M, Thouyaret L, Laudi R, Campanera A. Evaluation of scaling-up of HPV self-collection offered by community health workers at home visits to increase screening among socially vulnerable under-screened women in Jujuy Province, Argentina. Implementation Science. 2017;12(1):17. DOI: 10.1186/s13012-017$\underline{0548-1}$

8. Buist DSM, Tiro JA, Thayer C, Beatty T, Miglioretti DL, Lin J, et al. Improving the promise of embedded pragmatic trials: Surmountable barriers encountered in an evaluation of home-based HPV selfsampling to increase cervical cancer screening in overdue women. Contemp Clin Trials Commun. 2019. DOI: 10.1016/j. conctc. 2019.100413

9. Sultana F, English DR, Simpson JA, Brotherton JML, Drennan K, Mullins R, et al. Rationale and design of the iPap trial: a randomized controlled trial of home-based HPV selfsampling for improving participation in cervical screening by never- and underscreened women in Australia. BMC Cancer. 2014;14:207. DOI: 10.1186/1471-2407-14-207

10. Shin HY, Lee B, Hwang SH, Lee DO, Sung NY, Park JY, et al. Evaluation of satisfaction with three different cervical cancer screening modalities: clinician-collected Pap test vs. HPV test by self-sampling vs. HPV test by urine sampling. J Gynecol Oncol. 2019;30(5):e76. DOI: 10.3802/jgo.2019.30.e76

11. Peeters E, Cornet K, Cammu H, Verhoeven $\checkmark$, Devroey D, Arbyn M. Efficacy of strategies to increase participation in cervical cancer screening: GPs offering self-sampling kits for HPV testing versus recommendations 
to have a pap smear taken. A randomised controlled trial. Papillomavirus Research. 2020. DOI: 10.1016/j.pvr.2020.100194

12. Kamath Mulki A, Withers M. Human Papilloma Virus self-sampling performance in low- and middle-income countries. BMC Womens Health. 2021;21. DOI: 10.1186/s12905-02001158-4

13. Brewer N, Bartholomew K, Maxwell A, Grant J, McPherson G, Wihongi $\mathrm{H}$, et al. Comparison of two invitation-based methods for human papillomavirus (HPV) self-sampling with usual care among un- and under-screened Māori, Pacific and Asian women: study protocol for a randomised controlled community trial to examine the effect of self-sampling on participation in cervical-cancer screening. BMC Cancer. 2019;19(1):1198. DOI: 10.1186/ s12885-019-6401-y

14. Allen-Leigh B, Uribe-Zúñiga P, LeónMaldonado L, Brown BJ, Lörincz A, Salmeron J, et al. Barriers to HPV self-sampling and cytology among low-income indigenous women in rural areas of a middle-income setting: a qualitative study. BMC Cancer. 2017;17(1):734. DOI: 10.1186/s12885-0173723-5

15. De Melo Kuil L, Lorenzi AT, Stein MD, et al. The Role of Self-Collection by Vaginal Lavage for the Detection of HPV and HighGrade Intraepithelial Neoplasia. Acta Cytol. 2017;61 (6):425-433. DOI: 10.1159/000477331

16. Ajenifuja OK, Ikeri NZ, Adeteye OV, Banjo AA. Comparison between self-sampling and provider collected samples for Human Papillomavirus (HPV) Deoxyribonucleic acid (DNA) testing in a Nigerian facility. Pan Afr Med J. 2018;30:110. DOI: $10.11604 /$ pamj.2018.30.110.14321

17. Senkomago V, Ting J, Kwatampora J, et al. High-risk HPV-RNA screening of physicianand self-collected specimens for detection of cervical lesions among female sex workers in Nairobi, Kenya. Int J Gynaecol Obstet. 2018;143(2):217-224. DOI: 10.1002/ijgo.12628

18. Esber A, Norris A, Jumbe E, et al. Feasibility, validity and acceptability of self-collected samples for human papillomavirus (HPV) testing in rural Malawi. Malawi Med J. 2018;30(2):61-66. DOI: 10.4314/mmj.v30i2.2

19. Obiri-Yeboah D, Adu-Sarkodie Y, Djigma F, et al. Self-collected vaginal sampling for the detection of genital human papillomavirus (HPV) using careHPV among Ghanaian women. BMC Womens Health. 2017;17(1):86. DOI: $10.1186 / \mathrm{s} 12905-017-0448-1$

20. Kuriakose $S$, Sabeena S, Binesh D, Abdulmajeed J, Ravishankar N, Ramachandran A, et al. Diagnostic accuracy of self-collected vaginal samples for HPV DNA detection in women from South India. International Journal of Gynecology \&
Obstetrics. 2020;149(2):219-24. DOI: $10.1002 /$ ijgo.13116

21. Wong ELY, Cheung AWL, Huang F, Chor JSY. Can Human Papillomavirus DNA Self-sampling be an Acceptable and Reliable Option for Cervical Cancer Screening in Female Sex Workers? Cancer Nurs. 2018;41(1):45-52. DOI: $\underline{10.1097 /}$ NCC.0000000000000462

22. Cremer M, Maza M, Alfaro K, Morales Velado M, Felix J, Castle PE, et al. (2017). ScaleUp of a Human Papillomavirus Testing Implementation Program in El Salvador. Journal of lower genital tract disease, 21(1), 26-32. DOI: 10.1097/LGT.0000000000000280

23. Toliman PJ, Kaldor JM, Badman SG, et al. Evaluation of self-collected vaginal specimens for the detection of high-risk human papillomavirus infection and the prediction of high-grade cervical intraepithelial lesions in a high-burden, lowresource setting. Clin Microbiol Infect. 2019. DOI: $\underline{10.1016 / \mathrm{i} . \mathrm{cmi} .2018 .05 .025}$

24. Kelly H, Mayaud P, Segondy M, Pant Pai $\mathrm{N}$, Peeling RW. A systematic review and meta-analysis of studies evaluating the performance of point-of-care tests for human papillomavirus screening. Sex Transm Infect. 2017 Dec;93(S4):S36-S45. DOI: $10.1136 /$ sextrans-2016-053070

25. Arbyn M, Smith S B, Temin S, Sultana F, Castle P \& Collaboration on Self-Sampling and HPV Testing (2018). Detecting cervical precancer and reaching underscreened women by using HPV testing on self samples: updated meta-analyses. BMJ (Clinical research ed.), 363, k4823. DOI: 10.1136/bmj.k4823

26. Viviano M, Tran PL, Kenfack B, Catarino R, Akaaboune $M$, Temogne $L$, et al. Self- versus physician-collected samples for the follow-up of human papillomavirus-positive women in sub-Saharan Africa. Int J Womens Health. 2018; 10:187-194. DOI: 10.2147/IJWH.S154212

27. Buchanan Lunsford N, Ragan K, Lee Smith J, Saraiya M, Aketch M. Environmental and Psychosocial Barriers to and Benefits of Cervical Cancer Screening in Kenya. Oncologist. 2017;22(2):173-81. DOI: 10.1634/ theoncologist.2016-0213

28. Ampofo AG, Adumatta AD, Owusu E, Awuviry-Newton K. A cross-sectional study of barriers to cervical cancer screening uptake in Ghana: An application of the health belief model. PLOS ONE. 2020 (4):e0231459. DOI: 10.1371/journal.pone.0231459

29. Gottschlich A, Rivera-Andrade A, Grajeda E, Alvarez C, Mendoza Montano C, Meza R. Acceptability of Human Papillomavirus SelfSampling for Cervical Cancer Screening in an Indigenous Community in Guatemala. J Glob Oncol. 2017;3(5):444-454. DOl: 10.1200/ $\underline{\mathrm{JGO}} 2016.005629$ 
30. Gizaw M, Teka B, Ruddies F, Kassahun K, Worku D, Worku A, et al. (2020). Reasons for Not Attending Cervical Cancer Screening and Associated Factors in Rural Ethiopia. Cancer prevention research. 2020; 13(7), 593-600. DOI: 10.1158/1940-6207.CAPR-19-0485

31. Rashid S, Labani S, Das BC. Knowledge, Awareness and Attitude on HPV, HPV Vaccine and Cervical Cancer among the College Students in India. Natarajaseenivasan K, editor. PLoS ONE. 2016 11(11):e0166713. DOI: 10.1371/journal.pone.0166713

32. Akinlotan M, Bolin JN, Helduser J, Ojinnaka C, Lichorad A, McClellan D. Cervical Cancer Screening Barriers and Risk Factor Knowledge Among Uninsured Women. J Community Healt. 2017;42(4):770-8. DOI: $\underline{10.1007 /}$ s10900-017-0316-9

33. Mukama T, Ndejjo R, Musabyimana A, Halage AA, Musoke D. Women's knowledge and attitudes towards cervical cancer prevention: a cross sectional study in Eastern Uganda. BMC Women's Health. 2017;17(1):9. DOI: $10.1186 / \mathrm{s} 12905-017-0365-3$

34. Laskow B, Figueroa R, Alfaro KM, Scarinci IC, Conlisk E, Maza M, et al. A pilot study of community-based self-sampling for HPV testing among non-attenders of cervical cancer screening programs in El Salvador.
Int J Gynaecol Obstet. 2017;138(2):194-200. DOI: $10.1002 /$ ijgo.12204

35. Alfaro KM, Gage JC, Rosenbaum AJ, Ditzian $L R$, Maza M, Scarinci IC, et al. Factors affecting attendance to cervical cancer screening among women in the Paracentral Region of El Salvador: a nested study within the CAPE HPV screening program. BMC Public Health. 2015;15(1):1058. DOI: 10.1186/s12889-0152360-7

36. Yeh PT, Kennedy CE, de Vuyst H, Narasimhan M. Self-sampling for human papillomavirus (HPV) testing: a systematic review and metaanalysis. BMJ Glob Health. 2019;4(3):e001351. DOI: 10.1136/bmjgh-2018-001351

37. Murchland AR, Gottschlich A, Bevilacqua K, Pineda A, Sandoval-Ramírez BA, Alvarez CS, et al. HPV self-sampling acceptability in rural and indigenous communities in Guatemala: a cross-sectional study. BMJ Open. 2019;9(10):e029158. DOI: 10.1136/ bmjopen-2019-029158 